\title{
AN EQUIVALENCE INDUCED BY Ext AND Tor APPLIED TO THE FINITISTIC WEAK DIMENSION OF COHERENT RINGS
}

\author{
by ELWOOD WILKINS
}

(Received 5 August, 1996)

Let $R$ be a ring, see below for other notation. The functor categories (mod- $R, \mathrm{Ab}$ ) and $\left((R \text {-mod })^{\mathrm{op}}, \mathrm{Ab}\right)$ have received considerable attention since the $1960 \mathrm{~s}$. The first of these has achieved prominence in the model theory of modules and most particularly in the investigation of the representation theory of Artinian algebras. Both [11, Chapter 12] and [8] contain accounts of the use (mod- $R, A b)$ may be put to in the model theoretic setting, and Auslander's review, [1], details the application of $(\bmod -R, \mathrm{Ab})$ to the study of Artinian algebras. The category $\left((R \text {-mod })^{\text {op }}, \mathrm{Ab}\right)$ has been less fully exploited. Much work, however, has been devoted to the study of the transpose functor between $R$-mod and mod- $R$. Warfield's paper, [13], describes this for semiperfect rings, and this duality is an essential component in the construction of almost split sequences over Artinian algebras, see [4]. In comparison, the general case has been neglected. This paper seeks to remedy this situation, giving a con-

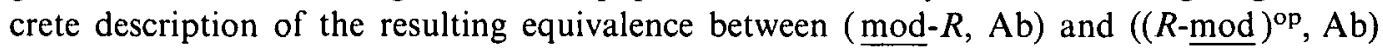
for an arbitrary ring $R$.

The first two sections are concerned with detailing an equivalence between $\left((R-\underline{m o d})^{\text {op }}\right.$, $\mathrm{Ab})$ and $(\bmod -R, \mathrm{Ab})$. The main result, Theorem 2.5 , states that the equivalence between these categories may be built from the functors Tor(-,-) and Ext(-,-). Parts of this theorem are well known. For instance the functor Tor(-,-) has been extensively studied in this setting when $R$ is an Artinian algebra, see [3]. In the third section this equivalence is applied to the Ziegler spectra of $R$. In Theorem 3.3, it is shown that the sets $\{M \mid f p$-inj.dim $M \leq n\}$ and $\{N \mid$ w.dim $N \leq n\}, n$ a natural number, are closed in their respective spectra when $R$ is left coherent. Furthermore, it is also demonstrated that these sets are mapped onto each other under Herzog's correspondence between the closed sets of the Ziegler spectra, [7, Theorem 5.5]. The main result of Section 3, Corollary 3.4, states that when $R$ is left coherent both spectra are test spaces for right finititistic weak dimension of $R$. Two special cases are noted: when $R$ is left Noetherian and when $R$ is left coherent and right perfect.

Throughout $R$ denotes an associative ring with unity. Mod- $R$ is the category of right $R$-modules, mod- $R$ the category of finitely presented right $R$-modules, $\underline{\bmod }-R$ is the quotient category of mod- $R$ modulo the ideal of those maps that factor through a projective. The left handed analogues of these categories are written as $R$-Mod, $R$-mod and $R$-mod, respectively. If $\mathcal{C}$ is a small additive category then $(\mathcal{C}, \mathrm{Ab})$ (respectively $\left(\mathcal{C}^{\mathrm{op}}, \mathrm{Ab}\right)$ ) is the category of covariant (respectively contravariant) additive functors from $\mathcal{C}$ to the category of abelian groups; $(\mathcal{C}, \mathrm{Ab})^{\mathrm{fp}}$ is the category of finitely presented objects of $(\mathcal{C}, \mathrm{Ab})$. If $F \in R$-mod and $G \in \bmod -R$ then $(-, F) \in\left((R-\bmod )^{\mathrm{op}}, \mathrm{Ab}\right)$ and $(G,-) \in(\bmod -R, \mathrm{Ab})$ are the corresponding projective objects.

All tensors are over $R$ and for $L, M \in R$-Mod, $N \in \operatorname{Mod}-R$, $\operatorname{Tor}(N, M)=\operatorname{Tor}_{1}^{R}(N, M)$ and $\operatorname{Ext}(L, M)=\operatorname{Ext}_{R}^{1}(L, M)$. If $M$ is a (left or right) $R$-module then w.dim $M$ is the weak dimension of $M$ and fp-inj.dim $M$ is the fp-injective dimension of $M$. Let $M \in R$-Mod, recall that w.dim $M$ is the least natural number $n$ such that $\operatorname{Tor}_{n+1}^{R}(-, M)=0$ and is infinite if no such natural number exists. The fp-injective dimension of $M$ is the least natural number $m$

Glasgow Math. J. 40 (1998) 167-176. 
such that $\operatorname{Ext}_{R}^{m+1}(F, M)=0$ for each $F \in R$-mod and is infinite if no such number exists. The fp-injective dimension is only well behaved over coherent rings; $R$ is left coherent if and only if, for each left $R$-module $M$, fp-inj.dim $M=m$ implies $\operatorname{Ext}_{R}^{m+k}(F, M)=0$ for each $F \in R$ mod and $k \geq 1$. Set r.fd $R$ to be the right finitistic weak dimension of $R$, that is r.fd $R=\sup \{w \cdot \operatorname{dim} N \mid N \in \operatorname{Mod}-R$, w.dim $N<\infty\}$, see [12] and [5].

The left Ziegler spectrum of $R$ is written as ${ }_{R} \mathrm{Zg}, \mathrm{Zg}_{R}$ denotes the right Ziegler spectrum. The reader is referred to [7] and [9] for the theory of the Ziegler spectrum in categorical setting employed in this paper, and to [14] for the original model theoretic exposition.

1. The functor Tor: $\left((\boldsymbol{R}-\mathbf{m o d})^{\circ}, \mathrm{Ab}\right) \rightarrow(\underline{\bmod }-\boldsymbol{R}, \mathbf{A b})$. Let $M$ be a left $R$-module; $(-, M)$ denotes the corresponding representable object of the category $\left((R \text {-mod })^{\mathrm{op}}, \mathrm{Ab}\right), \mathrm{P}(-, M)$ denotes the subobject of $(-, M)$ consisting of those homomorphisms that can be factored through a projective module and we set $(-, M)=(-, M) / P(-, M)$. For $H \in R$-mod and $x \in(H, M)$ it is easily shown that $x \in P(H, \bar{M})$ precisely when $x$ can be written as $x=\sum g_{i} y_{i}$, for some $g_{i}: H \rightarrow R$ and $y_{i} \in(R, M)$.

Lemma 1.1. Let $R^{m} \stackrel{W}{\longrightarrow} R^{n} \stackrel{w}{\longrightarrow} H \rightarrow 0$ be a presentation of $H \in R$-mod. Then, considering $(H, M)$ as a subgroup of $\left(R^{n}, M\right), x \in(H, M)$ if and only if $W x=0$ and $x \in \mathrm{P}(H, M)$ if and only if $x=V y$ for some $V \in\left(R^{n}, R^{p}\right)$ satisfying $W V=0$ and some $y \in\left(R^{p}, M\right)$.

Proof. The stated characterisation of $(H, M)$ is immediate from the exact sequence $0 \rightarrow(H, M) \rightarrow\left(R^{n}, M\right) \rightarrow\left(R^{m}, M\right)$. If $V \in\left(R^{n}, R^{p}\right)$ satisfies $W V=0$ then $V=w g$ for some $g: H \rightarrow R^{p}$. That $\operatorname{Im}(V, M) \subseteq P(H, M)$, under the given identification of $(H, M)$, follows. Let $x=\sum_{j=1}^{p} g_{i} y_{i} \in \mathrm{P}(H, M)$, where $g_{i}: H \rightarrow R, y_{i} \in(R, M)$, and let $v_{i}=w g_{i}$. Set $V: R^{n} \rightarrow$ $R^{p}$ to be the matrix whose i'th column is $v_{i}$, then $W V=W w \sum g_{i}=0$ and $w x=\sum w g_{i} y_{i}=$ $W\left(y_{i}\right)$ as required.

Let $f: F \rightarrow G \in \bmod -R$. Fix presentations $R^{a} \stackrel{U}{\longrightarrow} R^{b} \stackrel{u}{\longrightarrow} F \rightarrow 0, R^{c} \stackrel{v}{\longrightarrow} R^{d} \stackrel{\nu}{\longrightarrow} G \rightarrow 0$ and pick matrices $A, B$ which provide a commutative diagram

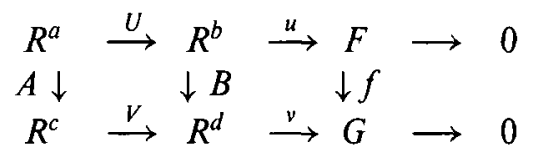

Set $U^{*}: R^{b} \longrightarrow R^{a} \in R$-mod etc. to be the $R$-dual of $U$ etc. and let

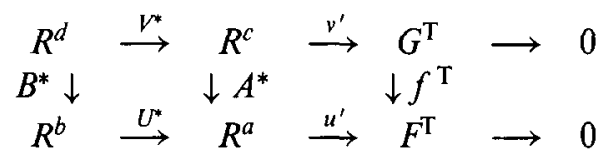

be the resulting diagram with exact rows between finitely presented left $R$-modules. The modules $F^{\mathrm{T}}$ etc. are called transposes of $F$ etc. and likewise the homomorphisms $f^{\mathrm{T}}$ etc. are called transposes of $f$ etc. 
It is convenient to work with matrices by considering their action on the bases of the free $R$-modules. Let

$$
\begin{aligned}
U: v_{i} & \mapsto \sum_{j} \pi_{j} u_{j i}, \quad V: \rho_{k} \mapsto \sum_{l} \sigma_{l} v_{l k}, \\
A: v_{i} & \mapsto \sum_{k} \rho_{k} a_{k i}, \quad B: \pi_{j} \mapsto \sum_{l} \sigma_{l} b_{l j}
\end{aligned}
$$

where $\left\{v_{i}\right\}$ etc. are the bases of the appropriate free right $R$-modulus, so that $\sum_{j} b_{l j} u_{j i}=\sum_{k} v_{l k} a_{k i}$ for each $i$ and $l$. All the above notation is fixed for the remainder of this section.

Let $M$ be a left $R$-module. There is an exact sequence $0 \rightarrow \operatorname{Tor}(F, M) \rightarrow$ $\operatorname{Im} U \otimes M \rightarrow R^{b} \otimes M$. We may thus identify $\operatorname{Tor}(F, M)$ with a subgroup of $\operatorname{Im} U \otimes M$ and as such

$$
\operatorname{Tor}(F, M)=\left\{\sum_{i}\left(\sum_{j} \pi_{j} u_{j i}\right) \otimes x_{i} \mid \sum_{i} u_{j i} x_{i}=0\right\}
$$

Using the identifications of Lemma 1.1, it is apparent that there is an epimorphism

$$
\begin{aligned}
t^{\prime}:\left(F^{\mathrm{T}}, M\right) & \rightarrow \operatorname{Tor}(F, M) \\
\left(x_{i}\right) & \mapsto \sum_{i}\left(\sum_{j} \pi_{j} u_{j i}\right) \otimes x_{i} .
\end{aligned}
$$

Let $U^{*} W=0, W y \in \mathrm{P}\left(F^{\mathrm{T}}, M\right)$ and write $W=\left(w_{i n}\right), y=\left(y_{n}\right)$. Then $t^{\prime}(W y)=\sum_{i}\left(\sum_{j} \pi_{j} u_{j i}\right) \otimes$ $\left(\sum_{n} w_{i n} y_{n}\right)=\sum_{n}\left(\sum_{j} \pi_{j} \sum_{i} u_{j i} w_{i n}\right) \otimes y_{n}=0$ and $\mathrm{P}\left(F^{\mathrm{T}}, M\right) \leq \operatorname{Ker} t^{\prime}$. Assume now that $\left(x_{i}\right) \in \mathrm{Ker} t^{\prime}$. From the exact sequence $\operatorname{Ker} U \otimes M \rightarrow R^{a} \otimes M \rightarrow \operatorname{Im} U \otimes M \rightarrow 0$, we have that $\sum_{i} v_{i} \otimes x_{i}=\sum_{n}\left(\sum_{i} v_{i} w_{i n}\right) \otimes y_{n}$, for suitable $\sum_{i} v_{i} w_{i n} \in \operatorname{Ker} U, y_{n} \in M$. Let $W=\left(w_{i n}\right)$, then $U^{*} W=0$ and $\left(x_{i}\right)=W\left(y_{n}\right)$. Thus $\mathrm{P}\left(F^{\mathrm{T}}, M\right)=\operatorname{Ker} t^{\prime}$. We have proved the following generalisation of [3, Proposition 2.2].

Lemma 1.2. For $F \in \bmod -R, M \in R-\operatorname{Mod}$ and any choice of $F^{\mathrm{T}}$, there is an isomorphism

$$
t_{F}^{M}: \underline{\left(F^{\mathrm{T}}, M\right)} \rightarrow \operatorname{Tor}(F, M) .
$$

Lemma 1.3. For $M \in R-$ Mod and any choice of the matrices in (1), the square

$$
\begin{array}{lll}
\frac{\left(F^{\mathrm{T}}, M\right)}{{\left(\sigma^{\mathrm{T}}, M\right)} \downarrow} & \stackrel{t_{F}^{M}}{\longrightarrow} & \operatorname{Tor}(F, M) \\
& \downarrow \operatorname{Tor}(f, M) \\
\underline{\left(G^{\mathrm{T}} M\right)} & \stackrel{t_{G}^{M}}{\longrightarrow} & \operatorname{Tor}(G, M)
\end{array}
$$

commutes. 
Proof. Let $x=\left(x_{i}\right)+\mathrm{P}\left(F^{\mathrm{T}}, M\right) \in \underline{\left(F^{\mathrm{T}}, M\right)}$, then

$$
\begin{aligned}
t_{G}^{M}\left(\underline{\underline{\left(f^{\mathrm{T}}, M\right)}}\right) & =\sum_{k}\left(\sum_{l} \sigma_{l} v_{l k}\right) \otimes\left(\sum_{i} a_{k i} x_{i}\right) \\
& =\sum_{i}\left(\sum_{l j} \sigma_{l} b_{l j} u_{j i}\right) \otimes x_{i} \\
& =\operatorname{Tor}(f, M)\left(\sum_{i}\left(\sum_{j} \pi_{j} u_{j i}\right) \otimes x_{i}\right) \\
& =\operatorname{Tor}(f, M)\left(t_{F}^{M} x\right) .
\end{aligned}
$$

Lemma 1.4. Let $g: M \rightarrow N \in R-\operatorname{Mod}$ and $x \in \underline{\left(F^{\mathrm{T}}, M\right)}$, then $f_{F}^{N}\left[\underline{\left(F^{\mathrm{T}}, g\right)} x\right]=\left(t_{F}^{M} x\right) \operatorname{Tor}(F, g)$.

Proof. Set $x=\left(x_{i}\right)+\mathrm{P}\left(F^{\mathrm{T}}, M\right)$, then

$$
\begin{aligned}
\left(t_{F}^{M} x\right) \operatorname{Tor}(F, g) & =\sum_{i}\left(\sum_{j} \pi_{j} u_{j i}\right) \otimes\left(x_{i} g\right) \\
& =t_{F}^{N}\left(\left(x_{i} g\right)+\mathrm{P}\left(F^{\mathrm{T}}, N\right)\right) \\
& =t_{F}^{N}\left[\underline{\left(F^{\mathrm{T}}, g\right)} x\right] .
\end{aligned}
$$

LEMmA 1.5. Let $M \in R$-Mod and $A \in\left((R-\bmod )^{\mathrm{op}}\right.$, Ab satisfy $\mathrm{P}(-, M) \leq A \leq(-, M)$. Define $T(A)(F)=\left\{t_{F}^{M} x \mid x \in A\left(F^{\mathrm{T}}\right) / \mathrm{P}\left(F^{\mathrm{T}}, M\right)\right\}$ and $T(A)(f): t_{F}^{M} x \mapsto t_{G}^{M}\left(x\left(f^{\mathrm{T}}, M\right)\right)$, then $T(A) \epsilon$ $\underline{\bmod }-R, \mathrm{Ab})$.

Proof. In the diagram (1), assume that $F=G$ and $f$ is the identity. Then $\operatorname{Tor}(f, M)$ : $t_{F}^{M} x \mapsto t_{G}^{M}\left(x\left(f^{\mathrm{T}}, M\right)\right)$ is an isomorphism. Since $t_{F}^{M} x \in T(A)(F)$ gives $t_{G}^{M}\left(x\left(f^{\mathrm{T}}, M\right)\right) \in T(A)(G)$, the restriction of $\operatorname{Tor}(f, M)$ to $T(A)$ is well defined and is also an isomorphism. Thus $T(A)(F)$ is independent of the choice of transpose of $F$. Likewise $T(A)(f)$ is independent of the choice of the transpose of $f$ and $T(A): \underline{\bmod }-R \rightarrow \mathrm{Ab}$ is a well defined map. It remains to verify that $T(A)$ is an additive functor. The required properties are inherited from Tor $(-, M)$.

Lemma 1.6. Let $g: M \rightarrow N \in R$-Mod and $\alpha: A \rightarrow B \in\left((R-\bmod )^{\mathrm{op}}\right.$, Ab) satisfy $\mathrm{P}(-, M)$ $\leq A \leq(-, M), P(-, N) \leq B \leq(-, N)$ and $A \rightarrow(-, M) \stackrel{(-, g)}{\rightarrow}(-, N)=A \stackrel{\alpha}{\rightarrow} B \rightarrow(-, N)$. Define $T(\alpha): T(A) \rightarrow T(B)$ by $T(\alpha)_{F}: t_{F}^{M} x \mapsto t_{F}^{N}\left[\left(F^{\mathrm{T}}, g\right) x\right]$. Then $T(\alpha): T(A) \rightarrow T(B) \in(\bmod -R, \mathrm{Ab})$.

Proof. As $\operatorname{Im}(A / \mathrm{P}(-, M) \rightarrow \underline{(-, M)} \stackrel{(-, g)}{\longrightarrow}(-, N) \leq B / \mathrm{P}(-, N)$, the restriction of Tor $(-, g)$ to $T(A)$ does give a map $T(\alpha): T(A) \rightarrow T(B)$. That $T(\alpha)_{F}$ is independent of the choice of transpose follows from Lemma 1.3 and that $T(\alpha)$ is a natural follows from the same lemma and Lemma 1.4. That $T(\alpha)$ is additive follows from the additivity of Tor $(-, g)$.

Let $\beta: C \rightarrow D \in\left((R-\underline{\bmod })^{\mathrm{op}}, \mathrm{Ab}\right)$. Construct a diagram

$$
\begin{array}{ccccc}
0 \rightarrow A & \rightarrow & (-, M) & \rightarrow & C \rightarrow 0 \\
\alpha \downarrow & & \downarrow(-g) & & \downarrow \beta \\
0 \rightarrow B & \rightarrow & (-, N) & \rightarrow & D \rightarrow 0
\end{array}
$$


with $M$ and $N$ pure-projective left $R$-modules. Clearly $g: M \rightarrow N$ and $\alpha: A \rightarrow B$ satisfy the hypotheses of Lemma 1.6. Define Tor $\left.:(R-\underline{\bmod })^{\circ}, \mathrm{Ab}\right) \rightarrow(\bmod -R, \mathrm{Ab})$ by $\operatorname{Tor}(C)$ $=\operatorname{Tor}(-, M) / T(A)$ and setting $\operatorname{Tor}(B)$ to be the unique map which makes the diagram

$$
\begin{array}{ccccc}
0 \rightarrow T(A) & \rightarrow & \operatorname{Tor}(-, M) & \longrightarrow & \operatorname{Tor}(C) \rightarrow 0 \\
T(\alpha) \downarrow & & \downarrow \operatorname{Tor}(-, g) & & \downarrow \operatorname{Tor}(\beta) \\
0 \rightarrow T(B) & \rightarrow & \operatorname{Tor}(-, N) & \longrightarrow & \operatorname{Tor}(D) \rightarrow 0
\end{array}
$$

commute.

THEOREM 1.7. $\operatorname{Tor}(\beta): \operatorname{Tor}(C) \rightarrow \operatorname{Tor}(D)$ is independent (up to isomorphism) of the choice of $g: M \rightarrow N$. Hence Tor $:\left((R-\underline{\bmod })^{\mathrm{op}}, \mathrm{Ab}\right) \rightarrow(\underline{\bmod }-R, \mathrm{Ab})$ is a well defined additive functor.

Proof. It is routine to check that $\operatorname{Tor}(C)$ is well defined up to isomorphism and that, having fixed $g, \operatorname{Tor}(\beta)$ is likewise well defined. That Tor is an additive functor follows.

2. The functor Ext: $(\underline{\bmod }-\boldsymbol{R}, \mathbf{A b}) \rightarrow\left((\boldsymbol{R}-\underline{\bmod })^{0 \mathrm{p}}, \mathbf{A b}\right)$. Let $A \in(\bmod -R, \mathrm{Ab})$, then $A$ is an object of (mod- $R, \mathrm{Ab})$ if and only if $A(P)=0$ for each projective $P \in \bmod -R$, or equivalently if and only if $A(R)=0$. It follows that each $A \in(\bmod -R, \mathrm{Ab})$ has a unique largest subobject, $\varepsilon A$, which is an object of (mod- $R$, Ab). If $\alpha: A \rightarrow B \in(\bmod -R, \mathrm{Ab})$, then $\varepsilon \alpha: \varepsilon A \rightarrow \varepsilon B$ is defined by restriction. Observe that $\varepsilon$ is a torsion radical; in particular $\operatorname{Hom}(\varepsilon A, B / \varepsilon B)=0$ for $A, B \in(\bmod -R$, Ab). If $g: M \rightarrow N \in R$-Mod, then we abbreviate $\varepsilon(-\otimes g): \varepsilon(-\otimes M) \rightarrow$ $\varepsilon(-\otimes N)$ to $\varepsilon g: \varepsilon M \rightarrow \varepsilon N$.

Assume that (1) is a commutative diagram in $R$-mod with exact rows. As before $U^{*}$ etc. are the $R$-duals of $U$ etc. and (2) denotes the induced diagram in mod- $R$ with exact rows. Again $F^{\mathrm{T}}, f^{\mathrm{T}}$ etc. are called transposes of $F, f$ etc. If $M \in R$-Mod and $x \in M^{a}$ then, abusing notation, $x$ also denotes the corresponding homomorphism $x: R^{a} \rightarrow M$.

Lemma 2.1. Let $M \in R$-Mod and $x \in M^{a}$, then $\left(u^{\prime} \otimes M\right) x \in \varepsilon M\left(F^{\mathrm{T}}\right)$ if and only if $\operatorname{Ker} U \leq \operatorname{Ker} x$.

Proof. Assume that $\operatorname{Ker} U \leq \operatorname{Ker} x$ and let $h: F^{\mathrm{T}} \rightarrow R$. Set $r^{*}=h u^{\prime}: R^{a} \rightarrow R$ and let $r=r^{* *}: R \rightarrow R^{a}$ be its $R$-dual. Then $h u^{\prime} U^{*}=0$ gives $r U=0$ and $r \in \operatorname{Ker} x$. Thus $(h \otimes M)$ $\left(u^{\prime} \otimes M\right) x=r x=0$ and $\left(u^{\prime} \otimes M\right) x \in \varepsilon M\left(F^{\mathrm{T}}\right)$. Conversely assume $\left(u^{\prime} \otimes M\right) x \in \varepsilon M\left(F^{\mathrm{T}}\right)$ and let $r \in \operatorname{Ker} U$. If $r^{*}: R^{a} \rightarrow R$ denotes the $R$-dual of $r: R \rightarrow R^{a}, r^{*} U^{*}=0$ gives $r^{*}=h u^{\prime}$ for some $h: F^{\mathrm{T}} \rightarrow R$. Then $r x=(h \otimes M)\left(u^{\prime} \otimes M\right) x=0$ and $\operatorname{Ker} U \leq \operatorname{Ker} x$.

Let $I=\operatorname{Im} U$ and let $R^{a} \stackrel{U}{\rightarrow} R^{b}=R^{a} \rightrightarrows I \leftrightarrow R^{b}$ be the epi-moni factorisation of $U$. Lemma 2.1 gives an epimorphism

$$
\begin{aligned}
e^{\prime}:(I, M) & \rightarrow \varepsilon M\left(F^{\mathrm{T}}\right) \\
g & \mapsto\left(u^{\prime} \otimes M\right)(\pi g)
\end{aligned}
$$


LEMMA 2.2. The map e' induces an isomorphism

$$
e_{F}^{M}: \operatorname{Ext}(F, M) \rightarrow \varepsilon M\left(F^{\mathrm{T}}\right)
$$

Proof. It is enough to show that $g \in \operatorname{Ker} e^{\prime}$ if and only if $g$ factors through . Certainly $e^{\prime}(\iota x)=\left(u^{\prime} \otimes M\right)(\pi \iota x)=\left(u^{\prime} U^{*} \otimes M\right) x=0$. Suppose $\left(u^{\prime} \otimes M\right) x=0$ then, by the right exactness of $-\otimes M, x=\left(U^{*} \otimes M\right) y=U y$ for some $y \in M^{b}$. Writing $x=\pi g: R^{a} \rightarrow M, \pi g=\pi \iota y$ and $g=\iota y$.

Lemma 2.3. Let $g: M \rightarrow N \in R$-Mod. Then the squares

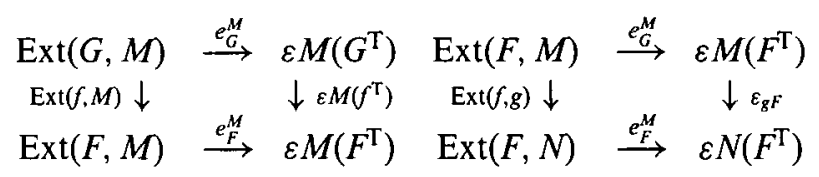

commute.

Proof. If $h: I \rightarrow M, \bar{h}$ denotes the corresponding element of $\operatorname{Ext}(F, M)$; a similar convention will be used for other Ext groups.

Let $R^{c} \stackrel{\pi^{\prime}}{\rightarrow} I^{\prime} \stackrel{l}{\prime}^{\prime} R^{d}$ be the epi-moni factorisation of $V$. The matrix $A$ induces a map $\alpha: I \rightarrow I^{\prime}$ which satisfies $\pi \alpha=A \pi^{\prime}$. If $h: I^{\prime} \rightarrow M$, then $\bar{h} \operatorname{Ext}(f, M)=\overline{(\alpha h)}$. We have

$$
\begin{aligned}
\varepsilon M\left(f^{\mathrm{T}}\right)\left[e_{G}^{M} \bar{h}\right] & =\left(f^{\mathrm{T}} v^{\prime} \otimes M\right)\left(\pi^{\prime} h\right) \\
& =\left(u^{\prime} \otimes M\right)(\pi \alpha h) \\
& =e_{F}^{M}[\bar{h} \operatorname{Ext}(f, M)]
\end{aligned}
$$

and the left hand square commutes.

If $h: I \rightarrow M$, then $\bar{h} \operatorname{Ext}(F, g)=\overline{(h g)}$. Thus

$$
\begin{aligned}
\left(e_{F}^{M} \bar{h}\right) \varepsilon g_{F^{\mathrm{T}}} & =\left[\left(u^{\prime} \otimes M\right) \pi h\right]\left(F^{\mathrm{T}} \otimes g\right) \\
& =\left(u^{\prime} \otimes N\right)(\pi h g) \\
& =e_{F}^{N}(\bar{h} \operatorname{Ext}(F, g))
\end{aligned}
$$

and the right hand square commutes as well.

Let $\alpha: A \rightarrow B \in(\underline{\bmod }-R, \mathrm{Ab})$; set $-\otimes M$ and $-\otimes N$ to be the respective injective envelopes of $A$ and $B$ in (mod- $R, \mathrm{Ab}$ ). Pick $g: M \rightarrow N$ such that $A \rightarrow-\otimes M \stackrel{-\otimes g}{\longrightarrow}-\otimes N=$ $A \stackrel{\alpha}{\rightarrow} B \rightarrow-\otimes N$. Since $\varepsilon \alpha=\alpha$, we have an induced commutative square

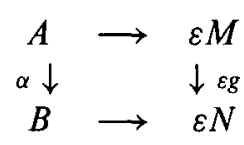

Define Ext $:(\underline{\bmod }-R, \mathrm{Ab}) \rightarrow\left((R-\underline{\bmod })^{o p}, \mathrm{Ab}\right)$ on objects by $\operatorname{Ext}(A)(F)=\left\{\bar{h} \mid e_{F}^{M} \bar{h} \in A\left(F^{\mathrm{T}}\right)\right\}$ and set $\operatorname{Ext}(\alpha): \operatorname{Ext}(A) \rightarrow \operatorname{Ext}(B)$ to be the restriction of $\operatorname{Ext}(-, g)$. 
THEOREM 2.4. Ext $:(\underline{\bmod }-R, \mathrm{Ab}) \rightarrow\left((R-\underline{\bmod })^{\mathrm{op}}, \mathrm{Ab}\right)$ is a well defined additive functor.

Proof. That $\operatorname{Ext}(A) \in\left((R-\underline{\bmod })^{\mathrm{op}},(\mathrm{Ab})\right.$ and that $\operatorname{Ext}(A)$ is independent (up to isomorphism) of the choice of $M$ is routinely verified. If $e_{F}^{M} \bar{h} \in A\left(F^{\mathrm{T}}\right)$, that $e_{F}^{N} \overline{(h g)} \in B\left(F^{\mathrm{T}}\right)$ is easily checked (using Lemma 2.3), and thus the definition of $\operatorname{Ext}(\alpha)$ makes sense. That Ext is a functor follows and the additivity of Ext is inherited from the additivity of $\operatorname{Ext}(-,-)$.

Theorem 2.5. Let $A \in\left((R-\underline{\bmod })^{\mathrm{op}}, \mathrm{Ab}\right), B \in(\underline{\bmod }-R, \mathrm{Ab})$. There are natural isomorphisms $A \cong \operatorname{Ext}(\operatorname{Tor}(A)), \operatorname{Tor}(\operatorname{Ext}(B)) \cong B$. Hence the pair $(\operatorname{Ext}$, Tor $):(\bmod -\mathrm{R}, \mathrm{Ab}) \rightarrow$ $\left((R-\underline{\mathrm{mod}})^{\mathrm{op}}, \mathrm{Ab}\right)$ is an equivalence.

Proof. We construct the natural isomorphism $\eta^{A}: A \rightarrow \operatorname{Ext}(\operatorname{Tor}(A))$, the isomorphism $\operatorname{Tor}(\operatorname{Ext}(B)) \cong B$ is left to the reader. Let $N \in R$-Mod be pure-projective and $0 \rightarrow C \rightarrow$ $(-, N) \stackrel{r}{\rightarrow} A \rightarrow 0$ exact. Let $-\otimes M$ be the injective envelope of $\operatorname{Tor}(A)$ and $\beta: \operatorname{Tor}(A) \rightarrow \varepsilon M$ the embedding. If $\gamma_{G} x \in A(G)$, then $t_{G}^{N}(x+\mathrm{P}(G, N))+T(C)\left(G^{\mathrm{T}}\right)=\left(u^{\prime} \otimes M\right) \pi^{\prime} h$ for some $f: I^{\prime} \rightarrow M$. Set $\eta_{G}^{A} \gamma_{G} x=\bar{h}$, which by definition is an element of $\operatorname{Ext}(\operatorname{Tor}(A))(G)$. Consider the following squares:

$$
\begin{aligned}
& A(G) \stackrel{\eta_{G}^{\Lambda}}{\longrightarrow} \operatorname{Ext}(\operatorname{Tor}(A))(G) \quad(G, N) \stackrel{\operatorname{Ext}(\beta)_{G G G}}{\longrightarrow} \quad \operatorname{Ext}(G, M) \\
& A() \downarrow \quad \downarrow \operatorname{Ext}(\operatorname{Tor}(A))(()) \quad(f, N) \downarrow \quad \downarrow \operatorname{Ext}(f, M) \\
& A(F) \stackrel{\eta_{F}^{A}}{\longrightarrow} \operatorname{Ext}(\operatorname{Tor}(A))(F) \quad(F, N) \stackrel{\operatorname{Ext}(\beta)_{F} F}{\longrightarrow} \operatorname{Ext}(F, M)
\end{aligned}
$$

The map $\gamma$ is an epimorphism and $\operatorname{Ext}(\beta)$ is monic (it is the restriction of $\operatorname{Ext}\left(-, 1_{M}\right)$ ). Thus to show that the left hand square commutes, it is enough to show that the right hand square commutes. Set $e_{F}=e_{F}^{M}, e_{G}=e_{G}^{M}$ and for $g \in(-, N)$ let $\underline{g}$ be the corresponding element of $\underline{(-, N)}$.

$$
\begin{aligned}
\left(\operatorname{Ext}(\beta)_{G G} g\right) \operatorname{Ext}(f, M) & =\left[\operatorname{Ext}(\beta)_{G}(g+C(G))\right] \operatorname{Ext}(f, M) \\
& =\left[e_{G}^{-1}\left(t_{G}^{N} \underline{g}+T(C)\left(G^{\mathrm{T}}\right)\right)\right] \operatorname{Ext}(f, M) \\
& =e_{F}^{-1}\left[\varepsilon M\left(f^{\mathrm{T}}\right)\left(t_{G}^{N} \underline{g}+T(C)\left(G^{\mathrm{T}}\right)\right)\right] \\
& =e_{F}^{-1}\left[\left(\operatorname{Tor}\left(f^{\mathrm{T}}, N\right) t_{G}^{N} g\right)+T(C)\left(F^{\mathrm{T}}\right)\right] \\
& =e_{F}^{-1}\left[t_{F}^{N}(f g)+T(C)\left(F^{\mathrm{T}}\right)\right] \\
& =\operatorname{Ext}(\beta)_{F}(f g+C(F)) \\
& =\operatorname{Ext}(\beta)_{F \gamma F}(f g) \\
& =\operatorname{Ext}(\beta)_{F \gamma F}(g(f, N)) .
\end{aligned}
$$

Therefore both squares commute and $\eta^{A}: A \rightarrow \operatorname{Ext}(\operatorname{Tor}(A)) \in\left((R-\underline{\bmod })^{\mathrm{op}}, \mathrm{Ab}\right)$. Since $A$ and $\operatorname{Ext}(\operatorname{Tor}(A))$ have isomorphic underlying groups and that the isomorphism between these groups is $\eta^{A}$, it follows that $\eta^{A}$ is a natural isomorphism. Finally it can be checked that if $\delta: A \rightarrow A^{\prime} \in\left((R \text {-mod })^{\mathrm{op}}, \mathrm{Ab}\right)$, then $\delta \eta^{A^{\prime}}=\eta^{A} \operatorname{Ext}(\operatorname{Tor}(\delta))$. This completes the verification that Ext $\circ$ Tor is isomorphic to the identity functor. 
3. The finitistic weak dimension. Recall, [14], that ${ }_{R} \mathrm{Zg}$ has points the indecomposable pure-injective left $R$-modules. By [8, Theorem B.16], we may consider the points of ${ }_{R} \mathrm{Zg}$ to be the indecomposable injective objects of $((\bmod -R), \mathrm{Ab})$. We shall switch between both formulations of the points of ${ }_{R} \mathrm{Zg}$ without comment. The closed sets of ${ }_{R} \mathrm{Zg}$ are in correspondence with the Serre subcategories of $(\bmod -R, \mathrm{Ab})^{\mathrm{fp}},[7$, Theorem 3.8] and [9, Corollary $2.10]$. Let $\mathcal{C}$ be a closed set of ${ }_{R} \mathrm{Zg}$ and $\mathcal{S}$ a Serre subcategory of (mod- $\left.R, \mathrm{Ab}\right)^{\mathrm{fp}}$, this correspondence is given by

$$
\begin{aligned}
& \mathcal{C} \mapsto\left\{A \in(\bmod -R, A b)^{\mathrm{fp}} \mid \operatorname{Hom}(A, \mathcal{C})=0\right\}, \\
& \mathcal{S} \mapsto \Sigma \mathcal{S}=\left\{M \in_{R} \mathrm{Zg} \mid \operatorname{Hom}(\mathcal{S},-\otimes M)=0\right\} .
\end{aligned}
$$

If $\mathcal{T}$ is a subset of $(\bmod -R, \mathrm{Ab})^{\mathrm{fp}},(\mathcal{T})$ denotes the smallest Serre subcategory of (mod- $\left.R, \mathrm{Ab}\right)^{\mathrm{fp}}$ containing $\mathcal{T}$.

Lemma 3.1. [7, Proposition 3.3]. If $\mathcal{T}$ is a subset of $(\bmod -R, \mathrm{Ab})^{\mathrm{fp}}$, then $\Sigma(\mathcal{T})=$ $\left\{M \in_{R} \mathrm{Zg} \mid \operatorname{Hom}(\mathcal{T},-\otimes M)=0\right\}$.

Auslander [2] and Gruson and Jensen [12] showed that there is a duality $D:$ (mod- $R$, $\mathrm{Ab})^{\mathrm{fp}} \rightarrow(R-\bmod , \mathrm{Ab})^{\mathrm{fp}}$ which is defined on objects by $D A(F)=\operatorname{Hom}(A,-\otimes F)$, $\left(F \in R\right.$-mod, $\left.A \in(\bmod -R, \mathrm{Ab})^{\mathrm{fp}}\right)$. This duality induces an order preserving bijection between the Serre subcategories of $(\bmod -R, \mathrm{Ab})^{\mathrm{fp}}$ and those of $(R-\bmod , \mathrm{Ab})^{\mathrm{fp}}$, [7, Theorem 5.5]. There is thus an equivalence between the closed sets of ${ }_{R} \mathrm{Zg}$ and $\mathrm{Zg}_{R}$. If $\mathcal{S} \subseteq(\bmod -R, \mathrm{Ab})^{\mathrm{fp}}$ is a Serre subcategory, then $\mathcal{D S}$ is the corresponding Serre subcategory of $(R \text {-mod, } \mathrm{Ab})^{\mathrm{f} \mathrm{p}}$, and if $\mathcal{C} \subseteq{ }_{R} \mathrm{Zg}$ is closed, then $\mathcal{C}^{*}$ denotes the corresponding closed subset of $\mathrm{Zg}_{R}$.

Proposition 3.2. $R$ is left coherent if and only if $\operatorname{Tor}(-, F) \in(\bmod -R, \mathrm{Ab})^{\mathrm{fp}}$ for each $F \in R$-mod. If $R$ is left coherent, $\operatorname{Tor}_{n}^{R}(-, F) \in(\bmod -R, \mathrm{Ab})^{\mathrm{fp}}$ for each $F \in R-\bmod$ and $n>0$.

Proof. Let $F \in R$-mod and fix a presentation $R^{a} \stackrel{U}{\longrightarrow} R^{b} \stackrel{u}{\rightarrow} F \rightarrow 0$. Let $R^{b} \stackrel{U^{*}}{\rightarrow} R^{a} \stackrel{u^{\prime}}{\longrightarrow}$ $F^{\mathrm{T}} \rightarrow 0$ be as before and $I=\operatorname{Im} U, J=\operatorname{Ker} U$. Construct the commutative diagram with exact rows:

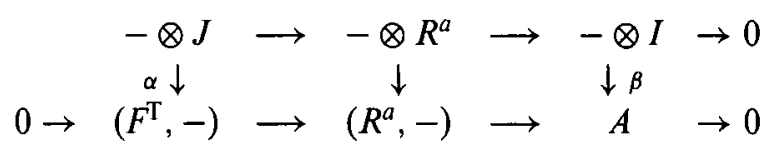

where $A=\operatorname{Ker}(-\otimes u)$. Since $\operatorname{Tor}(-, F)=\operatorname{Ker} \beta$ and $\beta$ is an epimorphism, there is an isomorphism $\operatorname{Tor}(-, F) \cong \operatorname{Cok} \alpha$. Thus $\operatorname{Tor}(-, F)$ is finitely presented if and only if $\operatorname{Im} \alpha$ is finitely generated if and only if $J$ is finitely generated if and only if $I$ is finitely presented. The first statement follows. For the second statement, note that when $R$ is left coherent and $n>1, \operatorname{Tor}_{n}^{R}(-, F) \cong \operatorname{Tor}(-, G)$ for some $G \in R$-mod.

TheOREM 3.3 [7, Example 5.1]. Let $R$ be a left coherent ring. For $n \geq 0$, set $\mathcal{X}_{n}=\left\{M \in{ }_{R} \mathrm{Zg} \mid \mathrm{fp}\right.$-inj.dim $\left.M \leq n\right\}$, and $\mathcal{Y}_{n}=\left\{N \in \mathrm{Zg}_{R} \mid\right.$ w.dim $\left.N \leq n\right\}$. Then $\mathcal{X}_{n}$ and $\mathcal{Y}_{n}$ are closed sets of their respective Ziegler spectra and $\mathcal{Y}_{n}=\mathcal{X}_{n}^{*}$. 
Proof. Let $F \in R$-mod, $M \in R$-Mod, $N \in \operatorname{Mod}-R$ and put $A=\operatorname{Tor}_{n+1}^{R}(-, F)$. We start by showing that $\operatorname{Ext}_{R}^{n+1}(F, M) \cong \operatorname{Hom}(A,-\otimes M)$ and $\operatorname{Tor}_{n+1}^{R}(N, F) \cong \operatorname{Hom}(D A, N \otimes-)$.

Set $n=0$. Using the equivalence of $\operatorname{Theorem~} 2.5, \operatorname{Ext}(F, M) \cong \operatorname{Hom}((-, F), \operatorname{Ext}(-, M)) \cong$ $\operatorname{Hom}((-, F), \operatorname{Ext}(-, M)) \cong \operatorname{Hom}(A, \varepsilon M) \cong \operatorname{Hom}(A,-\otimes M)$. Assume that $N$ is finitely presented, then $\operatorname{Tor}(N, F) \cong \operatorname{Hom}((N,-), A) \cong \operatorname{Hom}(D A, N \otimes-)$. For $N$ arbitrary, write $N=\lim _{\rightarrow} N_{i}$ with each $N_{i}$ finitely presented. Since $-\otimes-\operatorname{Tor}(-, F)$ and $\operatorname{Hom}(D A,-)$ all commute with direct limits, we have $\operatorname{Tor}(N, F) \cong \lim \operatorname{Tor}\left(N_{i}, F\right) \cong \lim \operatorname{Hom}\left(D A, N_{i} \otimes-\right)$ $\cong \operatorname{Hom}(D A, N \otimes-)$. For $n>0$, let $0 \rightarrow G \rightarrow P \longrightarrow F \rightarrow 0$ be exact with $P$ finitely generated projective. Then $\operatorname{Ext}_{R}^{n+1}(F, M) \cong \operatorname{Ext}_{R}^{n}(G, M) \cong \operatorname{Hom}\left(\operatorname{Tor}_{n}^{R}(-, G),-\otimes M\right) \cong \operatorname{Hom}(\mathrm{A},-\otimes \mathrm{M})$. Also $\operatorname{Tor}_{n+1}^{R}(N, F) \cong \operatorname{Tor}_{n}^{R}(N, G) \cong \operatorname{Hom}\left(D\left(\operatorname{Tor}_{n}^{R}(-, G)\right), N \otimes-\right) \cong \operatorname{Hom}(D A, N \otimes-)$.

Set $\mathcal{T}_{n}$ to be the Serre subcategory of $(\bmod -R, \mathrm{Ab})^{\mathrm{fp}}$ generated by the objects $\operatorname{Tor}_{n+1}^{R}(-, F)$ with $F \in R$-mod. By Lemma 3.1, fp-inj.dim $M \leq n$ if and only if $\operatorname{Hom}\left(\mathcal{T}_{n},-\otimes M\right)=0$ and w.dim $N \leq n$ if and only if $\operatorname{Hom}\left(\mathcal{D} \mathcal{T}_{n}, N \otimes-\right)=0$. The theorem follows from $\mathcal{X}_{n}=\Sigma \mathcal{T}_{n}$ and $\mathcal{Y}_{n}=\Sigma\left(\mathcal{D} \mathcal{T}_{n}\right)$.

We apply this theorem to the finitistic weak dimension of $R$. Krause [10, Theorem 1], has shown that for a two sided artinian ring, the finitistic projective dimension of the ring is the supremum of the projective dimension of the points in the Ziegler spectrum with finite projective dimension. The following result generalises this, the proof is analogous.

Corollary 3.4. If $R$ is left coherent then r.fd $R=\sup \left\{w \cdot \operatorname{dim} N \mid N \in \mathrm{Zg}_{R}, w \cdot \operatorname{dim} N\right.$ $<\infty\}=\sup \left\{\mathrm{fp}-\mathrm{inj} \cdot \operatorname{dim} M \mid M \in{ }_{R} \mathrm{Zg}, \mathrm{fp}\right.$-inj.dim $\left.M<\infty\right\}$.

Proof. If for some $n \geq 0$ and $K \in \operatorname{Mod}-R, w \cdot \operatorname{dim} K=n+1$ then $\operatorname{Hom}\left(\mathcal{T}_{n}, K \otimes-\right) \neq 0$, $\operatorname{Hom}\left(\mathcal{T}_{n+1}, K \otimes-\right)=0$. Thus $\mathcal{T}_{n}$ properly contains $\mathcal{T}_{n+1}$. Using the correspondence between Serre subcategories of $(R-\bmod , \mathrm{Ab})^{\mathrm{fp}}$ and closed subsets of $\mathrm{Zg}_{R}$, this gives that $\mathcal{X}_{n}$ is properly contained in $\mathcal{X}_{n+1}$. Thus if $N \in \mathcal{X}_{n+1} \backslash \mathcal{X}_{n}$,w.dim $N=n+1$. This proves the first equality. Recalling from [12, Proposition 3.4] that $\operatorname{r.fd} R=\sup \{\mathrm{fp}-\mathrm{inj} \cdot \operatorname{dim} M \mid M \in R-\mathrm{Mod}, \mathrm{fp}-$ $\operatorname{inj} \operatorname{dim} M<\infty\}$, the other equality is similarly proved.

Two special cases of this result are worth noting. If $R$ is left Noetherian, then the fpinjective dimension of a left module is its injective dimension. Thus the left Ziegler spectrum of a left Noetherian ring is a test space for the left finitistic injective dimension of $R$, see [5, Section 5]. The other case is when $R$ is a right perfect and left coherent ring. In this situation the weak dimension of a right module is its projective dimension, this following directly from Bass' Theorem P, [5], a part of which characterises right perfect rings as being those for which flat modules are projective. For such a ring, the above corollary states that the right Ziegler spectrum is a test space for the right finitistic projective dimension of $R$.

\section{REFERENCES}

1. M. Auslander, A functorial approach to representation theory, in Representations of algebras, eds. M. Auslander and E. Lluis, Lecture Notes in Mathematics 944 (Springer-Verlag, 1982), 105-179.

2. M. Auslander, Isolated Singularities and almost split sequences, in Representation Theory II, eds. V. Dlab, P. Gabriel, G. Michler, Lecture Notes in Mathematics 1178 (Springer-Verlag, 1987), 194242. 
3. M. Auslander and I. Reiten, Representation theory of Artin algebras III. Almost split sequences, Comm. Algebra 3 (1975), 239-294.

4. M. Auslander, I. Reiten and S. O. Smalø, Representation theory of Artin algebras, Cambridge Studies in Advanced Mathematics 36 (Cambridge University Press, 1995).

5. H. Bass, Finitistic dimension and a homological generalisation of semi-primary rings, Trans. Amer. Math. Soc. 95 (1960), 466-488.

6. L. Gruson et C. U. Jensen, Dimension cohomologiques reliees aux foncteurs lim $\stackrel{(i)}{\rightarrow}$, in Sém. d'Algebre, P. Dubreil et M.-P. Malliavin, eds, Lecture Notes in Mathematics 867, (Springer-Verlag, 1981), 234-294.

7. I. Herzog, The Ziegler spectrum of a locally coherent Grothendieck category, Proc. London Math. Soc. (3) 74 (1997), 503-509.

8. C. U. Jensen and H. Lenzing, Model theoretic algebra (Gordon and Breach, 1989).

9. H. Krause, The spectrum of a locally coherent category, J. Pure Appl. Algebra, 114 (1997), $259-271$.

10. H. Krause, Finitistic dimension and the Ziegler spectrum, Proc. Amer. Math. Soc., to appear.

11. M. Prest, Model theory and modules, LMS Lecture Notes Series (Cambridge University Press, 1988).

12. B. Stenström, Coherent rings and FP-injective modules, J. London Math. Soc. 2 (1970), 323-329.

13. R. B. Warfield Jnr., Serial rings and finitely presented modules, J. Algebra 37 (1975) 187-222.

14. M. Ziegler, Model theory of modules, Ann. Pure Appl. Logic 26 (1984), 149-213.

Department of Computer Science

UNIVERSITY OF BRISTOL

BRISTOL

ENGLAND
Present address:

Department of Computer Science

UNIVERSITY OF ESSEX

COLCHESTER CO4 3SQ

ENGLAND 